\title{
Psicólogo da Saúde no Hospital Geral: um Estudo sobre a Atividade e a Formação do Psicólogo Hospitalar no Brasil
}

Role of Health Psychologists in a General Hospital in Brazil: Examining Their Education and Work Activities

Psicólogo de la Salud en el Hospital General: un Estudio sobre la Actividad y la Formación del Psicólogo Hospitalario en Brasil

\section{Raquel Ayres de Almeida \& Lucia Emmanoel Novaes Malagris}

Universidade Federal do Rio de Janeiro

http://dx.doi.org/10.1590/1982-3703001312013 
Resumo: A Psicologia da Saúde é uma área em expansão no Brasil e no mundo, se revelando como um campo de muitas possibilidades de atuação para o psicólogo, sendo o trabalho no hospital uma delas. Conhecer o cenário atual no que se refere ao perfil do psicólogo hospitalar e as abordagens teórico-metodológicas utilizadas neste contexto pode contribuir para o desenvolvimento da área. O objetivo deste estudo é a realização de um levantamento do perfil profissional de psicólogos da saúde que exercem atividades em instituições hospitalares nacionais. Participaram da pesquisa 125 psicólogos da saúde que atuam em hospital geral e foi utilizado como instrumento o Questionário de Pesquisa Acadêmica e Profissional. Os resultados gerais mostram que a maioria se encontra na região sudeste $(62,4 \%)$, possui cursos de pós-graduação na área da saúde $(77,6 \%)$, trabalha em hospitais públicos $(70,4 \%)$, costuma participar de congressos e eventos científicos $(88 \%)$, atua no setor de internação (84\%) e ambulatório $(60 \%)$, enquanto as emergências parecem ainda "suspirar" pelo atendimento contínuo deste profissional (28\%). Quanto à clientela atendida, grande parte dos profissionais tem como foco de atuação no hospital tanto os pacientes (92,8\%), quanto a família (88\%) e a equipe (64\%). Diante desse perfil, conclui-se que a Psicologia Hospitalar parece se desenvolver de forma positiva no Brasil, observando-se que os profissionais estão buscando aprimoramento na área. Sabe-se, no entanto, que são necessários alguns avanços, principalmente no que tange à inserção deste profissional nas unidades de saúde do país.

Palavras-chave:Medicina doComportamento. Psicólogos. Educaçãoem Saúde. Credenciamento.

Abstract: Health psychology is a field that has been rapidly expanding in Brazil and throughout the world. It has proven to be an area with many possibilities for psychologists, and working in hospitals is just one of the many opportunities available. A clear understanding of the current profile of psychologists who work in hospitals as well as of the theoretical and methodological approaches used in this context can contribute to the development of the field. This study aimed to conduct a survey of the professional profile of health psychologists who work at Brazilian hospitals. One hundred and twenty-five health psychologists working in general hospitals participated in this research. The Academic and Professional Research Survey was used as an instrument to get a descriptive overview of the current health psychologists. Overall results indicate that the majority of the sample (62.4\%) was from the southeast region of Brazil. Furthermore, most of the participants were graduates in the field related to health $(77.6 \%)$, worked in public hospitals $(70.4 \%)$, used to participate in conferences and scientific events $(88 \%)$, and worked at inpatient care settings (84\%) and at outpatient clinics $(60 \%)$. Emergency rooms $(28 \%)$ still seem to lack the continuous care from these specific professionals. Concerning the population that these participants assisted, the majority of the professionals working at hospitals focus on treating patients $(92.8 \%)$, their family $(88 \%)$, and the hospital staff $(64 \%)$. Based on this profile, it can be concluded that hospital psychology seems to be positively developing in Brazil, as it can be noted that the professionals are in search for qualification in the area. However, it is clear that advances are necessary, especially regarding the insertion of these professionals into the health unities in Brazil.

Keywords: Behavioral Medicine. Psychologists. Health Education. Credentialing.

Resumen: La Psicología de la Salud es un área en gran expansión en Brasil y el mundo, revelándose como un campo de muchas posibilidades de actuación para el psicólogo, siendo una de ellas el trabajo en el hospital. Conocer el escenario actual, en lo que se refiere al perfil del psicólogo hospitalario y los abordajes teóricos y metodológicos utilizados en ese contexto, puede contribuir al desarrollo del área. Tuvimos como objetivo analizar el perfil profesional de psicólogos de la salud que ejercen actividades en instituciones hospitalarias nacionales. Participaron de la investigación 125 psicólogos de la salud que actúan en hospitales generales y se utilizó como instrumento el Cuestionario de Investigación Académica y Profesional. Los resultados generales señalan que la mayor parte de la muestra (el 62,4\%) está en la región sudeste, posee cursos de posgrado en el área de la salud (el 77,6\%), trabaja en hospitales 
públicos (el 70,4\%), suele participar en congresos y eventos científicos (el 88\%), actúa en el sector de la internación (el 84\%) y el ambulatorio (el 60\%), mientras las urgencias (el 28\%) parecen todavía suspirar por la atención continua de ese profesional. En cuanto al público que atienden, la mayoría de los profesionales tienen como foco de actuación en el hospital tanto los pacientes (el 92,8\%), como la familia (el 88\%) y el equipo (el 64\%). Ante este perfil, concluimos que la psicología hospitalaria parece desarrollarse de manera positiva en Brasil, observándose que los profesionales están buscando perfeccionamiento en el área. Sabemos, sin embargo, que son necesarios algunos progresos, principalmente en lo que atañe a la inserción de este profesional en las unidades de salud del país.

Palabras clave: Medicina de la Conducta. Psicólogos. Educación en Salud. Habilitación Profesional.

\section{Introdução}

No Brasil, as instituições de saúde constituem um campo de atuação para os psicólogos, sendo observado um crescente interesse e inserção do profissional nessa área. Esse crescimento se deve à necessidade de entender e intervir no processo saúde/doença de acordo com uma dimensão psicossocial e de compreender e intervir sobre os indivíduo ou grupos, expostos a diferentes doenças e condições de saúde impróprias (Almeida, \& Malagris, 2011).

A Psicologia da Saúde busca compreender o papel das variáveis psicológicas sobre a manutenção da saúde, o desenvolvimento de doenças e seus comportamentos associados. Os psicólogos da saúde realizam intervenções com o objetivo de prevenir doenças e auxiliar no manejo ou no enfrentamento das mesmas, além de desenvolver pesquisas (Almeida, \& Malagris, 2011; Miyazaki, Domingos, Caballo \& Valerio, 2011).

A maioria dos psicólogos da saúde trabalha em hospitais, clínicas e departamentos acadêmicos de faculdades e universidades. $\mathrm{Na}$ atuação clínica, podem fornecer atendimento para pacientes com dificuldades de ajustamento à condição de doente. Pode-se também ensinar aos pacientes métodos psicológicos para ajudá-los a manejar ou gerir os problemas de saúde, como aprender a controlar as condições de dor (Sarafino, 2004).

A intervenção em centros de saúde e hospitais deve abranger a tríade paciente - familiares profissionais de saúde. A família, angustiada e sofrida, também precisa da atenção do psicólogo e deve ser envolvida no trabaIho com o paciente por ser uma das raras motivações que este tem para enfrentar o sofrimento. O psicólogo deve facilitar, criar e garantir a comunicação efetiva e afetiva entre paciente/família e equipe. (Angerami-Camon, Trucharte, Knijnik, \& Sebastiani, 2006; Ismael, 2005; Romano, 1999). A equipe de saúde vivencia no seu cotidiano o significado de viver e morrer, além de sentimentos ambivalentes de onipotência e impotência, a cobrança da expectativa de todos os envolvidos e a percepção da própria finitude. $\mathrm{O}$ psicólogo deve atuar como facilitador do fluxo dessas emoções e reflexões, detectar os focos de stress e sinalizar as defesas exacerbadas (Angerami-Camon, 2002).

Segundo Teixeira (2004), os campos de atuação clínica do psicólogo da saúde podem incluir prestação de cuidados de saúde na atenção básica e de média complexidade, unidades de internação hospitalar (alta complexidade), serviços de saúde mental, unidades de dor, oncologia, consultas de supressão do tabagismo, serviços de reabilitação, entre outros. Em uma unidade hospitalar, o psicólogo da saúde pode prestar assistência no ambulatório clínico, nas unidades de emergência ou pronto-socorro, unidades de internação ou enfermarias e nas unidades e centros de terapia intensiva - UTI e CTI (Almeida, \& Malagris, 2011).

A Associação Americana de Psicologia (APA, 2010) demarca o trabalho do psicólogo em hospitais como um dos possíveis locais de atuação do psicólogo da saúde. No Brasil, o termo Psicologia Hospitalar tem sido utilizado 
para designar o trabalho de psicólogos em hospitais, sendo uma especialidade reconhecida pelo Conselho Federal de Psicologia (2010).

O presente estudo teve como objetivo realizar um levantamento do perfil profissional de psicólogos da saúde que atuam em instituições hospitalares no Brasil. Acredita-se que essas informações poderão contribuir para o conhecimento sobre o campo de atuação do psicólogo hospitalar à medida que identificará o panorama deste setor da Psicologia no país.

\section{Método}

Trata-se de uma pesquisa de levantamento realizada por meio de correio eletrônico para coleta de dados através de um questionário. Os participantes do estudo foram selecionados a partir de 16 grupos de discussão brasileiros sobre Psicologia da Saúde e a partir dos contatos das próprias autoras do estudo, totalizando 2.121 contatos. Destes, responderam a pesquisa 125 profissionais, que preencheram eletronicamente o Termo de Consentimento Livre e Esclarecido.

Foi utilizado como instrumento para realização do estudo o Questionário de Pesquisa Acadêmica e Profissional, QPAP. O questionário foi elaborado pelas autoras do estudo e é composto de perguntas fechadas a respeito da formação acadêmico-profissional e atividades desempenhadas na instituição. Como itens abordados, incluem-se o estado em que o profissional atua, tempo de atividade na área, tempo de formado em Psicologia, pós-graduação, participação em congressos e eventos científicos, participação em associações na área, tipo de hospital, nível de complexidade, tipo de vínculo com o hospital, setor de atuação, clientela, carga horária e referencial teórico.

O presente artigo refere-se à parte de uma pesquisa maior intitulada "Perfil e Prática Profissionais do Psicólogo da Saúde no Hospital Geral", que teve como objetivo realizar um levantamento do perfil profissional de psicólogos da saúde que exercem atividades em instituições hospitalares nacionais para verificar aqueles que utilizam como abordagem teórico-metodológica a terapia cognitivo-comportamental, com as principais técnicas utilizadas. Dessa forma, os dados apresentados são parciais e restritos aos aspectos relacionados à formação acadêmico-profissional e atividades desempenhadas pelo profissional.

A pesquisa completa foi aprovada pelo Comitê de Ética em Pesquisa da Escola de Enfermagem Anna Nery, protocolo $n^{\circ}$ 004/2011.

A análise dos dados foi realizada através de uma análise descritiva, com o objetivo de verificar a percentagem dos itens abordados entre os psicólogos atuantes em instituições hospitalares.

\section{Resultados e discussão}

Participaram do estudo 125 profissionais, sendo 112 mulheres $(89,6 \%)$ e 13 homens $(10,4 \%)$, como pode ser observado na Tabela 1. O número expressivo de participantes do sexo feminino condiz com diversas pesquisas na área de Psicologia. Yamamoto, Siqueira e Oliveira (1997) realizaram uma pesquisa no Rio Grande do Norte $(n=190)$ e observaram o enorme predomínio do sexo feminino $(88,4 \%)$ sobre o masculino $(11,6 \%)$ e se referiram à Psicologia como uma profissão essencialmente feminina. Dados nacionais do Conselho Federal de Psicologia (2004) referem uma diferença ainda maior: dentre os participantes da pesquisa com profissionais do país $(n=2000), 91 \%$ eram do sexo feminino e $9 \%$ masculino. Esses dados também estão de acordo com a colocação de Dimenstein (1998), que afirma que a Psicologia é marcada como uma profissão feminina.

Essa discrepância parece se repetir na área da Psicologia da Saúde. Oliveira et al. (2004), em sua pesquisa com psicólogos da rede básica de saúde em Natal, RN ( $n=28)$, encontraram números também expressivos: 93\% mulheres, ao passo que apenas $7 \%$ de homens. Do mesmo modo, Yamamoto, Trindade e Oliveira (2002) realizaram uma pesquisa com 25 psicólogos que atuavam em hospitais no Rio Grande do Norte, dentre 
Tabela 1. Dados Sociodemográficos e de Formação Acadêmica da amostra

\begin{tabular}{|c|c|c|c|}
\hline Itens & Categorias & Frequência & $\%$ \\
\hline \multirow[t]{2}{*}{ Gênero $(n=125)$} & Feminino & 112 & 89,6 \\
\hline & Masculino & 13 & 10,4 \\
\hline \multirow{5}{*}{$\begin{array}{l}\text { Região onde atua profissionalmente } \\
(\mathrm{n}=125)\end{array}$} & Sudeste & 78 & 62,4 \\
\hline & Sul & 20 & 16 \\
\hline & Nordeste & 15 & 12 \\
\hline & Norte & 06 & 4,8 \\
\hline & Centro-Oeste & 06 & 4,8 \\
\hline \multirow{6}{*}{$\begin{array}{l}\text { Ano de conclusão da formação em } \\
\text { Psicologia }(n=125)\end{array}$} & 2010 & 05 & 4 \\
\hline & 2006-2009 & 33 & 26,4 \\
\hline & $2000-2005$ & 35 & 28 \\
\hline & 1995-1999 & 15 & 12 \\
\hline & 1990-1994 & 13 & 10,4 \\
\hline & Antes de 1990 & 24 & 19,2 \\
\hline \multirow[t]{2}{*}{ Curso de pós-graduação $(n=125)$} & Sim & 118 & 94,4 \\
\hline & Não & 07 & 5,6 \\
\hline \multirow[t]{4}{*}{ Tipo de pós-graduação $(\mathrm{n}=118)$} & Especialização & 79 & 66,9 \\
\hline & Mestrado & 28 & 23,7 \\
\hline & Doutorado & 17 & 14,4 \\
\hline & Pós-doutorado & 1 & 0,8 \\
\hline \multirow{2}{*}{$\begin{array}{l}\text { Relacionado à área de Psicologia } \\
\text { hospitalar e da saúde }(\mathrm{n}=118)\end{array}$} & Sim & 97 & 82,2 \\
\hline & Não & 20 & 16,9 \\
\hline \multirow{4}{*}{$\begin{array}{l}\text { Participação em congressos e eventos } \\
\text { científicos nos últimos dois anos }(n=110)\end{array}$} & 1 evento & 20 & 18,2 \\
\hline & 2 a 5 eventos & 71 & 64,6 \\
\hline & 6 a 10 eventos & 15 & 13,6 \\
\hline & Mais de 10 eventos & 4 & 3,6 \\
\hline
\end{tabular}

eles $80 \%$ feminino e $20 \%$ masculino. Essa expressiva predominância do gênero feminino nas pesquisas pode estar associada à representação social da profissão de psicólogo como uma profissão feminina e, talvez, com algum preconceito por parte dos homens na escolha de uma profissão que já vem sendo associada ao gênero feminino.

Quanto à região de atuação profissional (Tabela 1), verificou-se que a maioria dos participantes atua na região sudeste $(62,4 \%)$, seguindo-se os participantes da região sul $(16 \%)$, região nordeste $(12 \%)$ e regiões norte e centro-oeste (ambas com 4,8\%). Esse dado pode ser decorrente do fato das autoras do estudo residirem na região sudeste e, portanto, possuírem mais contatos nestes estados. Contudo, em consulta à Sociedade Brasileira de Psicologia Hospitalar em 2009, as pesquisadoras foram informadas de que a região sudeste contempla o maior número de psicólogos associados (62,8\%), o que poderia indicar que a região possui um maior número de profissionais atuando na área hospitalar. Dados de 2009 do Instituto Brasileiro de Geografia e Estatística (2011) apontam que o sudeste é a região com maior número de instituições de saúde $(37,6 \%)$ e a região com maior quantidade de equipamentos de infraestrutura existentes em estabelecimentos de saúde (46,2\%). Acredita-se que esses dados se cruzam numa relação direta, ou seja, por ser uma região mais desenvolvida com recursos 
tecnológicos, o sudeste provavelmente oferece uma maior oferta de trabalho, atraindo mais profissionais da área da saúde. Contudo, cabe pensar e questionar se nas outras regiões, por serem menos desenvolvidas, a atuação do psicólogo hospitalar é valorizada, ou seja, é possível que algumas cidades com menos recursos não tenham esse profissional atuando. Sugere-se que novos estudos sejam realizados contemplando essa distribuição espacial dos profissionais.

Em relação ao tempo de formação em Psicologia, $41,6 \%$ dos participantes é formado há mais de 12 anos, enquanto 30,4\% tem até cinco anos e $28 \%$ tem entre seis e 11 anos de formado. A maior parte (32\%) dos profissionais atua em Psicologia Hospitalar há mais de 10 anos, enquanto 29,6\% tem entre um e quatro anos de atividade na área, $16,8 \%$ entre quatro e sete anos, $13,6 \%$ entre sete e 10 anos e $8 \%$ atua há menos de um ano (Tabela 2).

O tempo de formado dos participantes da pesquisa está coerente com o tempo de atuação em Psicologia Hospitalar: a maioria atua há mais de 10 anos e está formada há mais de 12, seguidos dos participantes que tem até cinco anos de formado e entre um e quatro anos de atuação. Esses dados sugerem que os profissionais iniciaram sua atuação na área hospitalar logo após concluírem a graduação, o que pode estar associado a oportunidades de estágio na área ou a realização de concurso logo após a formatura. Esse dado nos leva a pensar que, possivelmente, as universidades estão abrangendo o tema, possibilitando assim, um interesse por parte do aluno ainda na graduação, levando-o a ingressar na área logo após a formação. É possível ainda que alguns concursados sejam movidos pelo interesse na área da saúde, mas também podem estar interessados apenas numa estabilidade financeira, sem necessariamente se identificarem com a prática da Psicologia Hospitalar.

A maioria dos participantes possui curso de pós-graduação $(94,4 \%)$ dentre os quais, $82,2 \%$ dos cursos são relacionados à área de Psicologia Hospitalar e da Saúde. Dentre os profissionais que alegaram ter pós-graduação, $66,9 \%$ relatou ter o título de especialista, $23,7 \%$ de mestre, $14,4 \%$ de doutor e apenas um participante relatou possuir pós-doutorado (Tabela 1). Além disso, observou-se que 110 $(88 \%)$ dos profissionais participaram de congressos e eventos científicos nos últimos dois anos, dentre estes, 71 (64,6\%) participaram de dois a cinco eventos nesse intervalo de tempo, $20(18,2 \%)$ apenas de um evento no mesmo período, $15(13,6)$ entre seis a 10 e quatro $(3,6 \%)$ participaram de mais de 10 eventos. Convém ressaltar que 15 (12\%) não têm frequentado eventos científicos nos últimos dois anos na área (Tabela 1).

Verificou-se, portanto, que a maioria dos participantes possui curso de pós-graduação na área de Psicologia Hospitalar e da Saúde, participando de congressos e eventos científicos, revelando uma preocupação desses profissionais com a troca de experiência e aprimoramento, o que pode sugerir uma procura por atualização na área e mostra que o psicólogo hospitalar, em sua maioria, não está simplesmente transpondo o modelo psicoterápico clássico de consultório para o hospital, como refere Dimenstein (1998).

Esses dados estão de acordo com pesquisas que demonstram a procura dos profissionais de Psicologia por outras modalidades de formação, como os cursos de pós-graduação lato sensu em sua maioria (Yamamoto, Siqueira, \& Oliveira, 1997; Yamamoto, Trindade, \& Oliveira, 2002; Oliveira et al., 2004). Pode-se observar no presente estudo e nas pesquisas referidas que os cursos stricto sensu são preteridos em relação aos cursos lato sensu e às chamadas formações clínicas. De acordo com Yamamoto, Trindade e Oliveira (2002), a procura pelos cursos está relacionada à deficiência na formação da graduação. Em sua pesquisa, $70 \%$ dos entrevistados afirmaram que a qualidade dos cursos de graduação é insuficiente para a atuação no mercado de trabalho. Os cursos de pós-graduação de especialização costumam ter um com perfil mais prático do que um stricto sensu, por isso é possível que os primeiros sejam mais frequentemente escolhidos no sentido de virem a contribuir no aprimoramento profissional. 
Tabela 2. Distribuiç̧ão do perfil da amostra

\begin{tabular}{|c|c|c|c|}
\hline Itens & Categorias & Frequência & $\%$ \\
\hline \multirow[t]{5}{*}{ Tempo de atividade na área } & Até um ano & 10 & 8,0 \\
\hline & Mais de um até quatro anos & 37 & 29,6 \\
\hline & Mais de quatro até sete anos & 21 & 16,8 \\
\hline & Mais de sete até 10 anos & 17 & 13,6 \\
\hline & Mais de 10 anos & 40 & 32,0 \\
\hline \multirow[t]{2}{*}{ Tipo de hospital } & Público & 88 & 70,4 \\
\hline & Particular & 37 & 29,6 \\
\hline \multirow[t]{3}{*}{ Nível de complexidade } & Nível Terciário & 96 & 76,8 \\
\hline & Nível Secundário & 28 & 22,4 \\
\hline & Nível Primário & 01 & 0,8 \\
\hline \multirow[t]{8}{*}{ Tipo de vínculo com hospital } & Empregatício & 83 & 66,4 \\
\hline & Professor e/ou Supervisor & 13 & 10,4 \\
\hline & Prestação de Serviço & 12 & 9,6 \\
\hline & Pesquisador & 10 & 8,0 \\
\hline & Estágio & 09 & 7,2 \\
\hline & Temporário & 06 & 4,8 \\
\hline & Residência & 05 & 4,0 \\
\hline & Outro & 03 & 2,4 \\
\hline \multirow[t]{6}{*}{ Setor do hospital em que atua } & Internação & 105 & 84 \\
\hline & Ambulatório & 75 & 60 \\
\hline & UTI / CTI & 62 & 49,6 \\
\hline & Emergência & 35 & 28 \\
\hline & Recursos Humanos & 03 & 2,4 \\
\hline & Outro & 03 & 2,4 \\
\hline \multirow[t]{5}{*}{ Clientela } & Pacientes Internados & 116 & 92,8 \\
\hline & Familiares de Pacientes & 110 & 88 \\
\hline & Funcionários e Equipe de Saúde & 80 & 64 \\
\hline & Pacientes de Ambulatório & 73 & 58,4 \\
\hline & Outro & 02 & 1,6 \\
\hline \multirow[t]{5}{*}{ Carga horária de trabalho } & Até 10 h semanais & 05 & 4 \\
\hline & Entre 11 e $20 \mathrm{~h}$ semanais & 15 & 12 \\
\hline & Entre 21 e $30 \mathrm{~h}$ semanais & 46 & 36,8 \\
\hline & Entre 31 e $40 \mathrm{~h}$ semanais & 40 & 32 \\
\hline & Mais de $40 \mathrm{~h}$ semanais & 19 & 15,2 \\
\hline \multirow[t]{7}{*}{ Referencial teórico utilizado } & Psicanálise & 42 & 33,6 \\
\hline & Terapia Cognitivo-Comportamental & 32 & 25,6 \\
\hline & Psicoterapia Breve de base analítica & 29 & 23,2 \\
\hline & Existencial-Humanista & 07 & 5,6 \\
\hline & Gestalt Terapia & 04 & 3,2 \\
\hline & Sistêmico & 03 & 2,4 \\
\hline & Outro & 08 & 6,4 \\
\hline
\end{tabular}


No entanto, também se pode pensar que ainda existam mais cursos lato sensu do que stricto sensu na área da Psicologia da saúde no Brasil, o que pode explicar em parte os resultados encontrados.

Em contrapartida, dados do Conselho Federal de Psicologia (2004) mostram que apenas $58 \%$ dos profissionais que responderam a uma pesquisa de opinião com psicólogos inscritos no conselho possuíam cursos de pós-graduação, contra $42 \%$ de profissionais que relataram não possuir. Pode-se pensar que na área da Psicologia Hospitalar essa procura seja maior devido ao pouco oferecimento de disciplinas específicas da área nos cursos de graduação e de estágios disponíveis na área, levando os profissionais a procurarem uma formação complementar. De acordo com Chiattone (2000), a mera transposição do método de atendimento no consultório para o âmbito hospitalar pode ser desastrosa, uma vez que cada contexto tem objetivos e metodologias diferentes, delimitando a atuação do psicólogo. Dessa forma, ressalta-se a importância de uma formação complementar para os profissionais que pretendem ingressar na área da Psicologia da Saúde.

Quanto ao tipo de hospital (Tabela 2), 70,4\% dos profissionais participantes da pesquisa trabalhavam em hospitais públicos, enquanto $29,6 \%$ eram de hospitais particulares. O nível de complexidade dos hospitais foi identificado na maioria como nível terciário $(76,8 \%)$, seguido pelo nível secundário (22,4\%), e um participante alegou atuar em nível primário. Dessa forma, a maioria dos profissionais relatou trabalhar em hospitais públicos $(70,4 \%) \mathrm{e}$ de nível terciário (76,8\%). Outras pesquisas confirmam esses dados (Yamamoto, Siqueira, \& Oliveira, 1997; Yamamoto, Trindade, \& Oliveira, 2002), verificando números expressivos de profissionais trabalhando no setor público na área da saúde (59,5\% e 79\% respectivamente).

Esse dado revela a inserção na área da Psicologia da Saúde por meio de concursos públicos e parece demonstrar que as novas políticas de saúde do governo estão levando a que vagas para psicólogos na área hospitalar sejam abertas, o que parece refletir a importância da profissão na prevenção, controle e tratamento de doenças de modo geral. Porém, cabe refletir e questionar se essa realidade transparece algo que as pesquisadoras percebem em sua prática: os hospitais públicos possuem um número expressivo de vagas para psicólogos, enquanto os hospitais particulares se restringem muitas vezes a um ou dois profissionais, ou até mesmo nenhum, em sua grade de funcionários. É possível que a rede particular ainda visualize o psicólogo hospitalar como um "artigo de luxo", em termos de custos, não percebendo os benefícios que a sua atuação pode acrescentar.

Em relação ao tipo de vínculo com o hospital (Tabela 2), a maioria alegou ter vínculo empregatício, como já esperado. É importante ressaltar que $20,4 \%$ são professores e/ou supervisores de estágios em hospitais, o que pode indicar que há um número representativo de instituições de ensino oferecendo curso de Psicologia Hospitalar. Chama atenção que apenas $8 \%$ dos profissionais são pesquisadores, demonstrando que a pesquisa na área ainda é escassa.

A maioria dos profissionais alegou atuar na internação (84\%) e no ambulatório (60\%), como pode ser observado na Tabela 2, o que está de acordo com dados da pesquisa de Yamamoto, Trindade e Oliveira (2002) em hospitais do Rio Grande do Norte. Porém, o número de psicólogos participantes do estudo atuando nas unidades/centros de terapia intensiva (UTI e CTI) também é expressivo $(49,6 \%)$, contrapondo $16 \%$ da pesquisa dos referidos autores. O grande número de psicólogos atuando nas internações e nos centros de tratamento intensivo dos hospitais está coerente com o nível de complexidade das instituições referidas no estudo. A emergência parece ainda ser um setor do hospital com pouca inserção do psicólogo: apenas 28\% dos participantes alegaram exercer suas funções nas emergências dos hospitais.

Muitos psicólogos também atuam em ambulatório, mesmo a maioria dos hospitais sendo de nível terciário. Pode-se supor que a demanda por acompanhamento psicoterápico nestas instituições seja expressiva. Além disso, é 
possível que a organização referente à atendimento primário, secundário e terciário, na prática, seja de difícil implementação, o que faz com que os objetivos de organização de níveis de atenção criados pelo SUS nem sempre sejam atingidos (Mendes, 2011). Pode-se pensar também numa falta de estruturação dos serviços de Psicologia, com diversos profissionais atuando em diferentes setores simultaneamente, em vez de ter um profissional designado para determinado setor. No caso específico dos hospitais particulares com poucos profissionais, pode-se refletir ainda numa sobrecarga de trabalho, sendo apenas um ou dois profissionais para dar conta de toda a demanda hospitalar.

Quanto ao tipo de clientela atendida pelos profissionais do estudo, 92,8\% alegaram atender pacientes internados, $88 \%$ atendem os familiares dos pacientes, $64 \%$ a equipe de saúde e funcionários e 58,4\% pacientes em ambulatório (Tabela 2). Os números demonstram que a maioria dos psicólogos hospitalares atua no atendimento tanto aos pacientes quanto a seus familiares e equipe de saúde, o que condiz com a teoria e com o ideal da Psicologia Hospitalar. Romano (1999) e Angerami-Camon (2002) ressaltam que a família, assim como o paciente, se angustia, sofre e se assusta com a possibilidade da morte, também necessitando da atenção do psicólogo. Da mesma forma, a equipe de saúde também vivencia sentimentos ambivalentes de onipotência e impotência, sofre cobranças de todos os envolvidos e deve ser assistida pelo psicólogo.

No que se refere à carga horária de trabalho dos psicólogos, verificou-se que a maior parte trabalha entre 21 e 30 horas semanais $(36,8 \%)$ e 31 e 40 horas semanais (32\%) (Tabela 2). De acordo com o Conselho Federal de Psicologia (2004), 46\% dos psicólogos entrevistados gastam entre 21 e 40 horas semanais na atividade principal no exercício da profissão. Essa diferença possivelmente reflete uma maior carga horária de trabalho na área da saúde, em concordância com os concursos públicos que exigem o mínimo de 20 horas semanais.
Destaca-se, na Tabela 2, a distribuição de respostas sobre o referencial teórico-metodológico utilizado pelos participantes, destacando-se a psicanálise $(33,6 \%)$, a terapia cognitivo-comportamental $(25,6 \%)$ e a psicoterapia breve de base analítica $(23,2 \%)$. Dados do Conselho Federal de Psicologia (2004) apontam para a predominância de psicólogos que utilizam como referencial teórico autores da Psicanálise. Se considerarmos a psicoterapia breve (PB) de base analítica e a Psicanálise como tendo uma base teórica em comum, já que a referida PB foi lançada a partir da preocupação de alguns psicanalistas em encontrar formas de abreviar o sofrimento de seus pacientes, sendo, portanto, tratamentos de natureza psicológica de duração inferior à da psicanálise clássica (Gilliéron, 1986; Oliveira, 1999), as abordagens de base psicanalítica correspondem a 56,8\% dos profissionais do estudo.

Dimenstein (1998) afirma que o alto grau de popularização da psicanálise no país se deve à sua difusão maciça a partir da década de 70 criando uma "cultura psicanalítica" (p. 70). Outro fator que pode ter influência na predominância da abordagem psicanalítica na área corresponde ao fato de que a maioria dos concursos públicos para psicólogos na saúde restringe a referência bibliográfica à literatura psicanalítica, o que leva, em grande parte, os concursados a atuarem de acordo com essa abordagem. Seria interessante que as provas abrangessem várias abordagens teóricas, visto que a Psicologia Hospitalar em si, não se restringe a nenhuma abordagem específica.

Em relação à participação em alguma associação de Psicologia Hospitalar e da Saúde, $72,8 \%$ dos profissionais da amostra relataram não possuírem esse tipo de vinculação (Tabela 3). Dentre aqueles que fazem parte de alguma associação na área, $27,2 \%$ da amostra total, $82,4 \%$ são sócios da Sociedade Brasileira de Psicologia Hospitalar (SBPH), 5, 9\% da Sociedade Brasileira de Psico-Oncologia (SBPO) e os demais se distribuíram entre Sociedade Brasileira de Cardiologia (SBC), Sociedade Paranaense de Cardiologia (SPC), Associação Brasileira de Stress (ABS), Sociedade Brasileira de 
Tabela 3. Distribuição dos participantes em associações relacionadas à Psicologia Hospitalar e da Saúde

\begin{tabular}{lccc}
\hline Itens & Categorias & Frequência & \% \\
\hline Participação em alguma associação $(\mathrm{n}=125)$ & Não & 91 & 72,8 \\
& Sim & 34 & 27,2 \\
\hline Associações $(\mathrm{n}=34)$ & SBPH & 28 & 82,4 \\
& SBPO & 02 & 5,9 \\
& SBC & 01 & 2,9 \\
& SPC & 01 & 2,9 \\
& ABS & 01 & 2,9 \\
& SBRAPO & 01 & 2,9 \\
& SBNp & 01 & 2,9 \\
& SOCESP & 01 & 2,9 \\
\hline
\end{tabular}

Psicologia Oncológica (SBRAPO), Sociedade Brasileira de Neuropsicologia (SBNp) e Sociedade de Cardiologia do Estado de São Paulo (SOCESP), encontrando-se um participante em cada uma delas, como pode ser visto na Tabela 3. É importante ressaltar que os participantes tinham a opção de citar mais de uma associação, o que ocorreu com dois participantes. Dessa forma, apesar de 34 participantes relatarem possuir vinculação com algum tipo de associação, o total da frequência de respostas é 36 .

É importante ressaltar que $72,8 \%$ é um número bastante expressivo e algumas suposições podem ser feitas a partir desse dado: a anuidade cobrada pela maioria das associações pode ser um empecilho à adesão; algumas pessoas podem não entender quais são as atribuições das associações e quais benefícios teriam ao associar-se; e outras podem ter tido alguma experiência passada com alguma associação e não ter tido nenhum benefício direto na sua atuação.

Como mostrado anteriormente, a maioria dos profissionais pesquisados se encontra na região sudeste do país e, por isso, resolveu-se fazer uma análise mais detalhada dessa região quanto ao desenho teórico (Tabela 4). No estado do Rio de Janeiro, as abordagens de base psicanalítica têm predominância, $35,9 \%$, enquanto a existencial humanista corresponde a $7,7 \%$, e a TCC e a gestalt terapia 3,8\% cada uma. No estado de Minas Gerais, $100 \%$ dos participantes alegaram utilizar a psicanálise como referencial teórico, enquanto no estado de São Paulo as abordagens de base psicanalítica correspondem a apenas $10,3 \%$; o restante dos

Tabela 4. Relação frequência de referencial teórico por região

\begin{tabular}{lllllllllllll}
\hline Região & $\mathbf{1}$ & $\mathbf{\%}$ & $\mathbf{2}$ & $\mathbf{\%}$ & $\mathbf{3}$ & $\mathbf{\%}$ & $\mathbf{4}$ & $\mathbf{\%}$ & $\mathbf{5}$ & $\mathbf{\%}$ & $\mathbf{6}$ & $\mathbf{\%}$ \\
\hline $\begin{array}{l}\text { Amostra } \\
\text { total }\end{array}$ & 42 & 100 & 32 & 100 & 29 & 100 & 07 & 100 & 04 & 100 & 03 & 100 \\
Sudeste & 30 & 71,4 & 18 & 56,3 & 14 & 48,3 & 6 & 85,7 & 3 & 75 & 0 & 0 \\
Sul & 3 & 7,1 & 8 & 25 & 7 & 24,1 & 0 & 0 & 1 & 25 & 1 & 33,3 \\
Nordeste & 7 & 16,7 & 2 & 6,2 & 3 & 10,3 & 1 & 14,3 & 0 & 0 & 2 & 66,7 \\
Norte & 1 & 2,4 & 1 & 3,1 & 4 & 13,8 & 0 & 0 & 0 & 0 & 0 & 0 \\
Centro-Oeste & 1 & 2,4 & 3 & 9,4 & 1 & 3,5 & 0 & 0 & 0 & 0 & 0 & 0 \\
\hline
\end{tabular}

1: Psicanálise; 2: Terapia cognitivo-comportamental; 3: Psicoterapia breve de base analítica; 4: Existencial humanista; 5: Gestalt terapia; 6: Sistêmica. 
profissionais do estado relatou utilizar a TCC. Cabe ressaltar que a terapia sistêmica não foi citada como referencial teórico por nenhum profissional da região sudeste e não houve nenhum participante no estudo do estado do Espírito Santo.

Em um comparativo entre Rio de Janeiro e São Paulo, analisou-se que no estado do Rio de Janeiro a grande maioria dos profissionais da pesquisa utiliza a psicanálise como referencial teórico (25,6\%), enquanto no estado de São Paulo, a grande maioria utiliza a terapia cognitivo-comportamental (19,2\%), como pode ser observado na Tabela 5 . Observa-se que em algumas regiões certos profissionais, pelo tempo de atuação e relevância da atividade e da instituição na qual desenvolveram seu trabalho, se tornaram importantes fontes de influência para a formação e inserção de outros profissionais na área da saúde em sua região. Esse fato parece definir, em parte, a predominância de determinada abordagem em dada região.

\section{Conclusão}

A Psicologia Hospitalar é uma área da Psicologia desenvolvida recentemente e que tem atraído muitos profissionais. Como campo de entendimento e tratamento dos aspectos psicológicos em torno do adoecimento e internação, a Psicologia Hospitalar vem demonstrando sua importância através de estudos e trabalhos publicados, com um grande avanço no seu desenvolvimento nos últimos anos. Contudo, faz-se necessário conhecer o seu status quo no Brasil, para que se possa avaliar de que forma ela está se desenvolvendo.

A presença do psicólogo hospitalar em uma instituição de saúde é de suma importância para a promoção e a manutenção da saúde física e emocional dos pacientes, além da prevenção e o tratamento de doenças. Ao identificar e compreender os fatores emocionais que intervêm na saúde do paciente, o psicólogo colabora para o enfrentamento da doença e tratamento, diminuindo assim, o sofrimento do mesmo e de sua família, além de colaborar para a adesão ao tratamento e recuperação do paciente.

Um dos objetivos deste estudo foi fazer um levantamento do perfil profissional dos psicólogos hospitalares através de um levantamento sócio-demográfico-ocupacional. Pode-se concluir que, dentre os profissionais de Psicologia Hospitalar que participaram da pesquisa, a maioria se encontra na região sudeste, possui cursos de pós-graduação na área da saúde, trabalha em hospitais públicos e costuma participar de congressos e eventos científicos. Foi verificado ainda que a maior parte deles não participa de nenhuma associação de Psicologia Hospitalar e da saúde e atua no setor de internação e ambulatório, enquanto as emergências parecem ainda suspirar pelo atendimento contínuo deste profissional. Quanto à clientela atendida, grande parte dos profissionais tem como foco de atuação no hospital tanto os pacientes, quanto a família e a equipe, conforme orientado na literatura.

Tabela 5. Comparativo entre Referenciais Teóricos em SP e RJ

\begin{tabular}{lcccccc}
\hline & \multicolumn{2}{c}{ Total } & \multicolumn{2}{c}{ São Paulo } & \multicolumn{2}{c}{ Rio de Janeiro } \\
\cline { 2 - 7 } Sudeste & Frequência & \% & Frequência & \% & Frequência & $\%$ \\
\hline Psicanálise & 42 & 100 & 02 & 2,6 & 20 & 25,6 \\
$\begin{array}{l}\text { Terapia } \\
\text { Cognitivo-Comportamental }\end{array}$ & 32 & 100 & 15 & 19,2 & 03 & 3,8 \\
$\begin{array}{l}\text { Psicoterapia Breve de base } \\
\text { analítica }\end{array}$ & 29 & 100 & 06 & 7,7 & 08 & 10,3 \\
$\begin{array}{l}\text { Existencial-Humanista } \\
\text { Gestalt Terapia }\end{array}$ & 07 & 100 & 0 & 0 & 06 & 7,7 \\
Sistêmico & 04 & 100 & 0 & 0 & 03 & 3,8 \\
\hline
\end{tabular}


Diante desse perfil, pode-se concluir que a Psicologia Hospitalar parece estar se desenvolvendo de forma positiva no Brasil, observando-se que os profissionais estão buscando aprimoramento na área. Sabe-se, no entanto, que são necessários alguns avanços, principalmente no que tange à inserção deste profissional nas unidades de saúde do país. Ainda que exista um projeto de lei federal que defende a obrigatoriedade da presença de psicólogo hospitalar em serviços de saúde públicos e privados, nem todos os hospitais contam com um psicólogo em sua rede de atendimento. Considerando a rede privada de saúde, quando há psicólogo contratado, se resume a um número simbólico de profissionais, sendo que a maioria dos psicólogos hospitalares se encontra na rede pública de saúde, como referido acima.

Visto que a atuação em hospitais não está vinculada a nenhuma teoria específica da Psicologia, podemos encontrar profissionais utilizando diversas abordagens teórico-metodológicas e pesquisar quais seriam as abordagens mais utilizadas no ambiente hospitalar foi outro objetivo do estudo. Concluiu-se que as teorias de base analítica, como psicanálise e psicoterapia breve, são as mais utilizadas entre os profissionais pesquisados, seguidas da terapia cognitivo-comportamental (TCC). 


\section{Raquel Ayres de Almeida}

Doutoranda pela Universidade Federal do Rio de Janeiro, Rio de Janeiro - RJ. Brasil.

E-mail: psi_raquel@yahoo.com.br

\section{Lucia Emmanoel Novaes Malagris}

Doutora pela Universidade Federal do Rio de Janeiro, Rio de Janeiro - RJ. Brasil.

E-mail: lucianovaes@terra.com.br

Endereço para envio de correspondência:

Rua Visconde de Pirajá, 282/1101. Ipanema. CEP: 20240-000. Rio de Janeiro - RJ. Brasil.

Recebido: 15/05/2013, $1^{\text {a }}$ Reformulação: 09/09/2014, Aprovado: 03/07/2015. 
Referências
Almeida, R. A.; \& Malagris, L. E. N. (2011). A prática da psicologia da saúde. Revista SBPH, 14(2), 183-202.

Angerami-Camon, V. A. (Org.). (2002). Psicologia da saúde: um novo significado para a prática clínica. São Paulo, SP: Pioneira.

Angerami-Camon, V. A., Trucharte, F. A. R., Knijnik, R. B., \& Sebastiani, R. W. (Orgs.). (2006). Psicologia hospitalar: teoria e prática. São Paulo, SP: Pioneira.

American Psychological Association - APA. (2010). Vancouver: o autor. Recuperado em 13 de março de 2010, de http://www.healthpsych.org/

Conselho Federal de Psicologia - CFP. (2004). Pesquisa de opinião com psicólogos inscritos no Conselho Federal de Psicologia. Brasília, DF: o autor. Recuperado em 3 de novembro de 2011, de http://www.pol. org.br/pol/cms/pol/publicacoes/relatorios/ relatorios_050513_0102.html

Conselho Federal de Psicologia - CFP. (2010). Resolução CFP 013/2007, Consolidação das Resoluções relativas ao Título Profissional de Especialista em Psicologia. Recuperado em 12 de março de 2010, de http://site.cfp.org. br/wp-content/uploads/2008/08/Resolucao_ CFP_nx_013-2007.pdf

Chiattone, H. B. C. (2000). A significação da psicologia no contexto hospitalar. In V. A. Angerami-Camon (Org.), Psicologia da saúde: um novo significado para a prática clínica (pp. 73-167). São Paulo, SP: Pioneira Psicologia.

Dimenstein, M. D. B. (1998). O psicólogo nas Unidades Básicas de Saúde: desafios para a formação e atuação profissionais. Estudos de Psicologia, 3(1), 53-81. doi:10.1590/S1413-294X1998000100004

Gilliéron, E. (1986). As psicoterapias breves. Rio de Janeiro, RJ: Jorge Zahar.

Instituto Brasileiro de Geografia e Estatística - IBGE. (2011). Pesquisa de assistência médico-sanitária 2009. Rio de Janeiro: o autor. Recuperado em 3 de novembro de 2011, de http://www.ibge.gov.br/home/ estatistica/populacao/condicaodevida/ ams/2009/default.shtm

Ismael, S.M.C. (2005). A inserção do psicólogo no contexto hospitalar. In S. M. C. Ismael (Org.), A prática psicológica e sua interface com as doenças (pp. 17-36). São Paulo, SP: Casa do Psicólogo.

Mendes, E. V. (2011). O sistema único de saúde: um processo social em construção. Recuperado em 07 de novembro de 2011: http://www.opas.org.br/rh/publicacoes/ textos_apoio/ACF9371.pdf

Miyazaki, M. C. O. S., Domingos, N. M., Caballo, V. E., \& Valerio, N. I. (2011). Psicologia da saúde: Intervenções em hospitais públicos. In B. Rangé (Org.), Psicoterapias cognitivocomportamentais: um diálogo com a psiquiatria (2a ed., pp.568-580). Porto Alegre, RS: Artmed.

Oliveira, I. F., Dantas, C. M. B., Costa, A. L. F., Silva, F. L., Alverga, A. R., Carvalho, D. B. et al. (2004). O psicólogo nas unidades básicas de saúde: formação acadêmica e prática profissional. Interações, 9(17), 71-89.

Oliveira, I. T. (1999). Psicoterapia psicodinâmica breve: dos precursores aos modelos atuais. Psicologia: Teoria e Prática, 1(2), 9-19.

Romano, B. W. (1999). Princípios para a prática da psicologia clínica em hospitais. São Paulo, SP: Casa do Psicólogo.

Sarafino, E. P. (2004). Context and perspectives in health psycology. In S. Sutton, A. Baum, \& M. Johnston, The Sage handbook of health psychology (pp. 1-26). London: Thousand Oaks.

Teixeira, J. A. C. (2004). Psicologia da saúde. Análise Psicológica, 3(22), 441-448.

Yamamoto, O. H., Siqueira, G. S., \& Oliveira, S. C. C. (1997). A psicologia no Rio Grande do Norte: caracterização geral da formação acadêmica e do exercício profissional. Estudos de Psicologia, 2(1), 42-67.

Yamamoto, O. H., Trindade, L. C. B. O., \& Oliveira, I. F. (2002). O psicólogo em hospitais no Rio Grande do Norte. Psicolologia USP, 13(1), 217-246. doi:10.1590/S0103-65642002000100011 